\title{
Subnanotesla Magnetometry with a Fiber-Coupled Diamond Sensor
}

\author{
R.L. Patel®, ${ }^{1,2, *}$ L.Q. Zhou $\odot,{ }^{1}$ A.C. Frangeskou $\odot,{ }^{1, \dagger}$ G.A. Stimpson $\odot,{ }^{1,2}$ B.G. Breeze $\odot,{ }^{1,3}$ \\ A. Nikitin $\odot,{ }^{1, \$}$ M.W. Dale ${ }^{1,}{ }^{\S}$ E.C. Nichols $\odot,{ }^{1}$ W. Thornley $\odot,{ }^{1}$ B.L. Green $\odot,{ }^{1}$ M.E. Newton, ${ }^{1,2}$ \\ A.M. Edmonds, ${ }^{4}$ M.L. Markham, ${ }^{4}$ D.J. Twitchen,${ }^{4}$ and G.W. Morley ${ }^{1,2,}{ }^{1}$ \\ ${ }^{1}$ Department of Physics, University of Warwick, Gibbet Hill Road, Coventry CV4 7AL, United Kingdom \\ ${ }^{2}$ EPSRC Centre for Doctoral Training in Diamond Science and Technology, University of Warwick, \\ Coventry CV4 7AL, United Kingdom \\ ${ }^{3}$ Spectroscopy RTP, University of Warwick, Gibbet Hill Road, Coventry CV4 7AL, United Kingdom \\ ${ }^{4}$ Element Six Innovation, Fermi Avenue, Harwell Oxford, Didcot OX11 OQR Oxfordshire, United Kingdom
}

(Received 3 March 2020; revised 11 September 2020; accepted 14 September 2020; published 30 October 2020)

Nitrogen-vacancy centers (NVCs) in diamond are being explored for future quantum technologies, and in particular ensembles of NVC are the basis for sensitive magnetometers. We present a fiber-coupled NVC magnetometer with an unshielded sensitivity of $(310 \pm 20) \mathrm{pT} / \sqrt{\mathrm{Hz}}$ in the frequency range of $10-150 \mathrm{~Hz}$ at room temperature. This takes advantage of low-strain ${ }^{12} \mathrm{C}$ diamond, lenses for fiber coupling and optimization of microwave modulation frequency, modulation amplitude, and power. Fiber coupling means the sensor can be conveniently brought within $2 \mathrm{~mm}$ of the object under study.

DOI: 10.1103/PhysRevApplied.14.044058

\section{INTRODUCTION}

The sensing of magnetic fields using the nitrogenvacancy center (NVC) in diamond has seen rapid growth over the last decade due to the promise of high-sensitivity magnetometry with exceptional spatial resolution [1,2] along with a high dynamic range [3]. The use of NVC ensembles rather than single centers improves the sensitivity while degrading the spatial resolution [3-13]. Recent advancements have demonstrated ensemble sensitivities of $0.9 \mathrm{pT} \sqrt{\mathrm{s}}$ for dc fields [14] and $0.9 \mathrm{pT} / \sqrt{\mathrm{Hz}}$ for ac fields [15]. However, these results have been limited to systems that are bulky and are typically fixed to optical tables. In contrast, fiber coupling provides a small sensor head that may be moved with relative independence from the rest

\footnotetext{
*rajesh.patel@warwick.ac.uk

${ }^{\dagger}$ Current address: Lightbox Jewelry, Orion House, 5 Upper St. Martin's Lane, London WC2H 9EA, United Kingdom.

$\$$ Current address: School of Engineering, University of Warwick, Coventry CV4 7AL, United Kingdom.

${ }^{\S}$ Current address: De Beers Group Technology, Belmont Rd, Maidenhead SL6 6JW, United Kingdom.

Igavin.morley@warwick.ac.uk

Published by the American Physical Society under the terms of the Creative Commons Attribution 4.0 International license. Further distribution of this work must maintain attribution to the author(s) and the published article's title, journal citation, and DOI.
}

of the control instrumentation and thus offers the possibility of application in medical diagnostic techniques such as magnetocardiography (MCG) [16-18].

Most fiber-coupled diamond magnetometers have relied on using nanodiamonds or microdiamonds attached to the end of a fiber, achieving sensitivities in the range of 56000-180 nT/ $\sqrt{\mathrm{Hz}}$ [19-21]. Utilizing a two-wire microwave transmission line in addition to a fiber-diamond setup was able to achieve a sensitivity of approximately $300 \mathrm{nT} / \sqrt{\mathrm{Hz}}$ [22]. A fiber-based gradiometer approach was able to provide a sensitivity of approximately $35 \mathrm{nT} / \sqrt{\mathrm{Hz}}$ with projected shot-noise sensitivities potentially allowing for MCG [23,24]. Using a hollowcore fiber with many nanodiamond sensors in a fluidic environment provided a sensitivity of $63 \mathrm{nT} / \sqrt{\mathrm{Hz}}$ per sensor and a spatial resolution of $17 \mathrm{~cm} \mathrm{[25].} \mathrm{Other} \mathrm{compact}$ magnetometers that use a fiber have demonstrated sensitivities in the ranges of $67-1.5 \mathrm{nT} / \sqrt{\mathrm{Hz}}$ [26-28]. The best sensitivity reported for a fiber-coupled diamond magnetometer so far is $35 \mathrm{nT} / \sqrt{\mathrm{Hz}}$ when sensing a real test field [23], and $1.5 \mathrm{nT} / \sqrt{\mathrm{Hz}}$ when estimating the sensitivity based on the signal to noise to linewidth using the slope of a resonance in the magnetic resonance spectrum [28]. Other diamond magnetometers, which offer high portability whilst maintaining a compact structure, have been demonstrated with a compact light-emitting-diode- (LED) based design achieving a minimum detectable field of $1 \mu \mathrm{T}$ whilst offering minimal power consumption [29].

Here, a diamond-based fiber-coupled magnetometer with sub-nT sensitivity is presented [30]. Though fiber 
coupling makes efficient optical excitation and detection more difficult a key feature of the sensor head is the use of lenses to reduce optical losses from the fiber to the diamond and back as shown in Fig. 1(b). The sensor head is very mobile being at the end of a 5-m optical fiber and the rest of the equipment is a $0.44 \times 0.55 \times 0.6 \mathrm{~m}^{3}$ box on wheels, which we successfully operate outside of our lab for demonstrations at trade shows. Though we currently are not sensitive enough to perform MCG, the configuration is well suited to this with no need for magnetic compensation coils.

The NVC, when in its negative charge state, is a spin $S=1$ defect that can be optically initialized into the $m_{s}=0$ ground state and possesses spin-dependent fluorescence giving rise to optically detected magnetic resonance (ODMR) [31,32]. The energy-level diagram is shown in Fig. 1(a). The Zeeman-induced splitting of the NVC leads to the detection of magnetic fields with high sensitivity where the magnetometer sensitivity is improved, by virtue of statistical averaging, when a larger number of centers are interrogated [33,34]. The zero-field splitting at room temperature is approximately $2.87 \mathrm{GHz}$. Upon application of an external magnetic field Zeeman-induced splitting leads to sublevels that are split by

$$
\Delta f=\frac{2 g_{e} \mu_{B} B_{\|}}{h},
$$

where $g_{e}=2.0028$ is the NVC $g$ factor, $\mu_{B}$ is the Bohr magneton, $B_{\|}$is the projection of the external magnetic field onto the NVC symmetry axis (the $\langle 111\rangle$ crystallographic direction) and $h$ is Planck's constant. The energy levels are further split by the hyperfine interaction between the electron spin and ${ }^{14} \mathrm{~N}$ nuclear $\operatorname{spin}(I=1)$ by $A \approx$
2.16 MHz. Under a continuous-wave excitation scheme, which is employed in this paper, the photon-shot-noiselimited sensitivity of a diamond-based magnetometer is given by

$$
\eta=\frac{4}{3 \sqrt{3}} \frac{h}{g_{e} \mu_{B}} \frac{\Delta v}{C \sqrt{I_{0}}},
$$

where $\Delta v$ is the linewidth, $C$ is the measurement contrast (the reduction in fluorescence when on resonance compared to when not on resonance), and $I_{0}$ is the number of photons collected per second $[5,35]$.

As demonstrated by Eq. (2), the quality of the diamond material is highly relevant to have high magnetic sensitivity. It is desirable to have a high NVC concentration to increase $I_{0}$, which reduces the photon-shot-noise limit. However, at high NVC concentrations there are significant magnetic interactions between spins $[36,37]$ and the sensitivity is degraded because of the increased NVC ODMR linewidth this causes. Furthermore, in practise there are always more substitutional nitrogen defects $\left(\mathrm{N}_{s}^{0}\right)$ than negatively charged NVC. These $\mathrm{N}_{s}^{0}$ defects also increase the NVC ODMR linewidth so an optimal NVC concentration is estimated to be around $1-10 \mathrm{ppm}[36,38]$. The conversion of $\mathrm{N}_{s}^{0}$ to $\mathrm{NVC}$ is also highly relevant and ideally would reach $50 \%$. The use of isotopically purified ${ }^{12} \mathrm{C}$ diamond further reduces the NVC ODMR linewidth because ${ }^{13} \mathrm{C}$ has a nuclear spin [39].

\section{METHODS}

Magnetometry is performed with the setup shown in Fig. 1(b). A Laser Quantum Gem-532 with a maximum
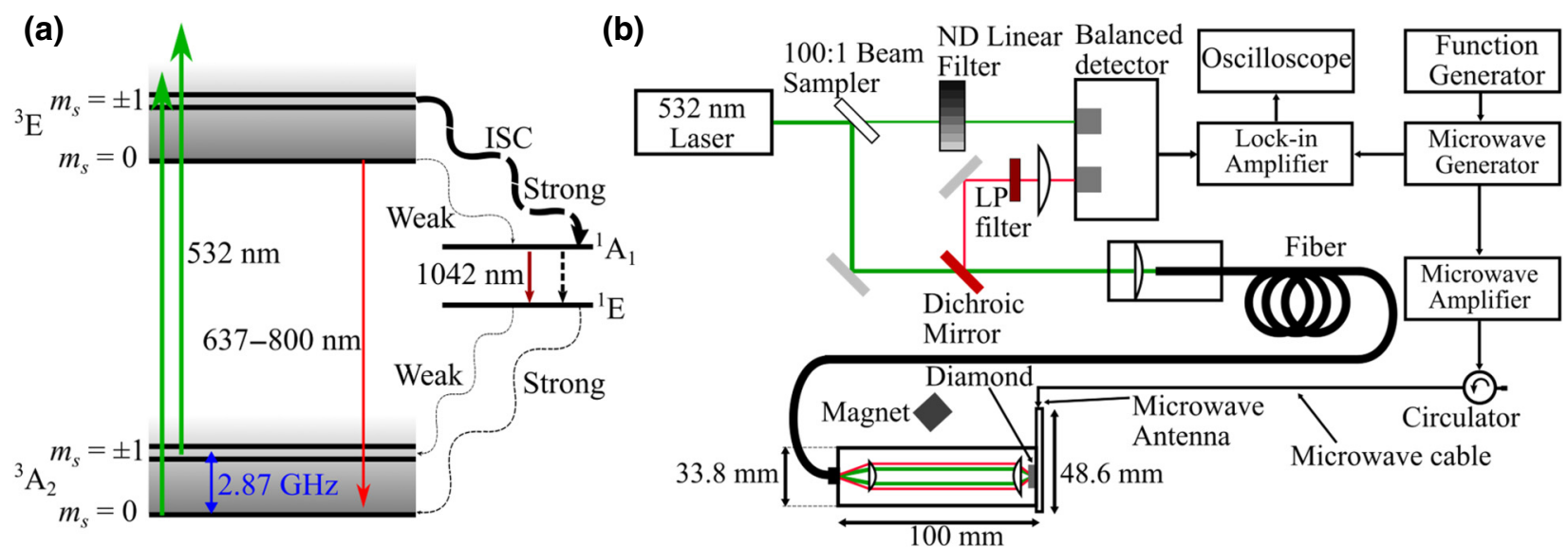

FIG. 1. (a) Energy-level diagram of the NVC in diamond, radiative and nonradiative transitions are indicated by solid lines and dashed lines, respectively. A 532-nm laser excites the system from the ${ }^{3} \mathrm{~A}_{2}$ states to the ${ }^{3} \mathrm{E}$ states. The $m_{s}= \pm 1$ states are more likely than the $m_{s}=0$ state to decay via the intersystem crossing (ISC) leading to spin polarization into the $m_{s}=0$ state. The ${ }^{3} \mathrm{E}$ state can decay back to the ${ }^{3} \mathrm{~A}_{2}$ by emitting 637 - to 800 -nm light and the intensity is greater for the $m_{s}=0$ state allowing for optical detection of magnetic resonance. (b) Schematic of our fiber-coupled magnetometer. The abbreviations ND and LP are neutral density and long pass, respectively. The dimensions of the sensor head are shown but other components are not to scale. 
power output of $2 \mathrm{~W}$ is used to excite the NVC ensemble; for our experiments $1 \mathrm{~W}$ is used to reduce laser noise. The laser beam is passed through a Thorlabs BSF10-A beam sampler whereby approximately $1 \%$ is picked off and supplied to the reference arm of a Thorlabs PDB450A balanced detector to cancel out laser-intensity noise; the illumination levels incident upon each photodiode is equal when resonant microwaves are applied and provides up to a factor of 11 enhancement in the sensitivity. The remaining (high-intensity) portion of the laser beam is focused into a custom-ordered 5-m 0.22 N.A. Thorlabs FG400AEA fiber with a core diameter of $400 \mu \mathrm{m}$ and ceramic FC/PC termination. Large movements to or bending or straining the fiber causes modal noise [40]: the variations in the collected photons on short timescales, which manifests as noise in our magnetometry. The fiber output is focused onto the diamond using a pair of aspheric lenses (Thorlabs) housed in a adjustable SM1 tube lens (Thorlabs SM1NR05). The first lens (C171TMD-B) collimates the fiber output whilst the second (C330TMD-B) focuses the beam onto the diamond. The same lenses are used to collect the emitted fluorescence from the NVC ensemble (see Appendix D).

Microwave excitation is provided by an Agilent N5172B microwave source with a Mini-Circuits ZHL$16 \mathrm{~W}-43-\mathrm{S}+$ microwave amplifier; hyperfine excitation is utilized to improve the contrast by mixing a $2.158 \mathrm{MHz}$ sinewave [5] from a RSPro arbitrary function generator AFG21005. The microwaves are then delivered to a $2-4-\mathrm{GHz}$ coaxial circulator to reduce microwave reflection. The microwaves are square-wave frequency modulated [41]. The signal from the balanced detector is supplied to a Zurich MFLI DC $-500 \mathrm{kHz}$ lock-in amplifier (LIA).
The diamond is mounted on a 2-mm diameter copper loop deposited onto an aluminum prototyping board (C.I.F AAT10) for microwave delivery and heat management. The thickness of the aluminum prototyping board is approximately $1.7 \mathrm{~mm}$, which limits how close the diamond can be brought to a magnetic field source. A permanent rare-earth magnet is aligned to the (111) crystallographic orientation of the NVC ensemble, providing the spectrum shown in Fig. 2(a). As per Eq. (1) the frequency splitting is $\Delta f \sim 114 \mathrm{MHz}$ yielding an applied magnetic field of approximately $2 \mathrm{mT}$. To enhance the sensitivity all three hyperfine resonances of the nitrogen-14 $\left(m_{I}=-1,0,1\right)$ are simultaneously excited by mixing the carrier wave with a $2.158-\mathrm{MHz}$ sinewave. This increases the number of observed resonances from three to the five shown for each resonance group.

The optimum sensitivity is determined by varying the microwave power, the modulation amplitude, and the frequency modulation to find the maximum value of the zero-crossing slope, as shown in Fig. 2. The microwave power is varied from -25 to $-8 \mathrm{dBm}$ (preamplification value before the $43-\mathrm{dB}$ amplifier, corresponding to 0.06 and $3.16 \mathrm{~W}$, respectively, after amplification with losses due to the cables neglected) in 1-dBm increments. The frequency modulation is investigated between 1 to $80 \mathrm{kHz}$ and the frequency modulation amplitude is varied from 100 to $600 \mathrm{kHz}$. All ODMR spectra are taken with a frequency sweep range of 2.76 to $2.94 \mathrm{GHz}$, a step resolution of $20 \mathrm{kHz}$, a step dwell time of $4 \mathrm{~ms}$ and a LIA frequency bandwidth of $200 \mathrm{~Hz}$ using a $48 \mathrm{~dB}$ /octave filter slope; the LIA output scaling was set to 500 .

The microwave frequency is then set to the zerocrossing point and the fluorescence is monitored with the sensitivity determined by considering the mean and
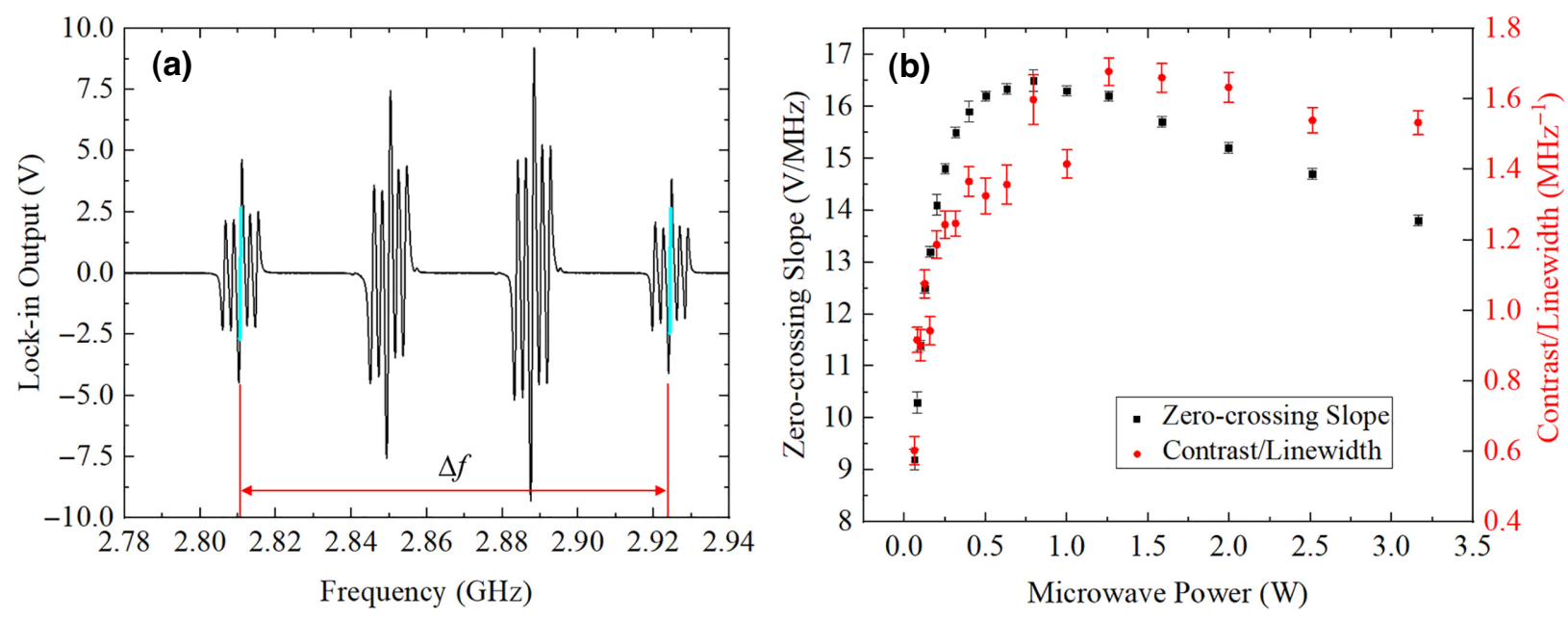

FIG. 2. (a) An ODMR spectrum of the NVC as a function of varying microwave frequency. (b) The zero-crossing slope and the contrast-to-linewidth ratio as a function of applied microwave power after amplification and neglecting cable losses. All measurements are taken using a frequency modulation amplitude of $300 \mathrm{kHz}$ and a modulation frequency of $3.0307 \mathrm{kHz}$. 
standard deviation of 160 1-s fast-Fourier transforms (FFTs) using an oscilloscope. The sample employed in this work is a single crystal $99.995 \%{ }^{12} \mathrm{C}$-enriched sample of dimension $4 \times 4 \times 0.6 \mathrm{~mm}^{3}$ with a (100) orientation, grown through chemical vapor deposition by Element Six [42]. The concentration of defects (see Appendix C) is determined using Fourier-transform infrared spectroscopy and ultraviolet-visible spectroscopy with the negatively charged NVC measured to be $4.6 \mathrm{ppm}$ [43-45]. Conversely, electron paramagnetic resonance (EPR) measurements indicate a concentration of $(2.8 \pm 0.2) \mathrm{ppm}$ for negatively charged NVC. The linewidth of an EPR spectrum is $0.46 \mathrm{MHz}$, which is in close agreement with the lowest ODMR linewidth we are able to achieve, $0.5 \mathrm{MHz}$, using a low microwave excitation power of $80 \mathrm{~mW}$ and in the absence of hyperfine excitation. The ODMR contrast at this microwave excitation power is $0.6 \%$. Pulsed EPR measurements yielded $T_{2}^{*} \sim 730 \mathrm{~ns}$.

\section{RESULTS}

An ODMR spectrum of the NVC when aligned along (111) is shown in Fig. 2(a). The microwaves are swept across the range specified in Sec. II, under this alignment only one of the four orientations of the NVC contribute to the magnetometry measurements; the other three NVC resonances are degenerate and thus occur at the same frequency. Two methods are used to determine the sensitivity. The first method involved fits to the outermost NVC lock-in derivative spectrum resonances, see Fig. 2(a), the fits are performed around the linear region of the ODMR resonances and this produced a calibration constant to convert the LIA output into one of magnetic field sensitivity. The other method involved applying known test fields to determine the magnetometer response. It is found that the maximum value of the zero-crossing slope occurred at a microwave power of approximately $0.8 \mathrm{~W}$, see Fig. 2(b), a frequency modulation amplitude of $300 \mathrm{kHz}$ and a modulation frequency of $1.0307 \mathrm{kHz}$. From Eq. (2) it can be inferred that the shot-noise-limited sensitivity is improved by increasing the ratio of contrast to linewidth $(C / \Delta v)$, but there is a trade-off here as shown in Fig. 2(b) because increasing the microwave power can increase both the contrast and the linewidth. To determine the translation of the $C / \Delta v$ and its relation to the optimum performance of the LIA output used for magnetometry, for a given set of microwave delivery parameters both a LIA output spectrum and the ODMR spectrum prior to lock-in amplification are taken; both are taken when exciting all three nitrogen-14 hyperfine resonances. It is found that the overall trends for both are highly correlated as expected however, at higher microwave powers we find that the sensitivity becomes worse beyond a microwave power of $0.8 \mathrm{~W}$; this is inferred from a decrease in the zero-crossing slope, which overall follows the trend of a decreasing $C / \Delta v$ ) ratio. Though the overall trend is followed there are discrepancies suggesting further refinement of the microwave parameters used in order to optimize the sensitivity. It is found that the zero-crossing slope increased with a decreasing modulation frequency $[5,41,46]$ (see Appendix A), however, beyond approximately $3 \mathrm{kHz}$ the noise floor of the FFT also increased and thus the best sensitivity is found to be $171 \mathrm{pT} / \sqrt{\mathrm{Hz}}$ at a modulation frequency of $3.0307 \mathrm{kHz}$. The value of the zero-crossing slope is $(17.9 \pm 0.2) \mathrm{V} / \mathrm{MHz}$ yielding a calibration constant of $5 \times 10^{-4} \mathrm{~V} / \mathrm{nT}$ when using the gyromagnetic ratio, $28 \mathrm{~Hz} / \mathrm{nT}$, of the NVC. This value improves upon the $1.5 \mathrm{nT} / \sqrt{\mathrm{Hz}}$ obtained with a fibercoupled diamond magnetometer with the same technique for sensitivity measurement.

For the second method known test fields are applied using a Helmholtz coil, which is calibrated using a Hirst Magnetics GM07 Hall probe. The test fields are applied along (100) and the sensitivity is found to be $(310 \pm$ 20) $\mathrm{pT} / \sqrt{\mathrm{Hz}}$, as shown in Fig. 3. The calibration constant in this instance is found to be $2.77 \times 10^{-4} \mathrm{~V} / \mathrm{nT}$ and is calculated by applying a linear fit to the data inset in Fig. 3. The noise at low frequencies, i.e., the $1 / f$ noise is attributed primarily to magnetic noise originating from the environment whilst the $50 \mathrm{~Hz}$ and harmonics thereof are attributed to magnetic noise from the mains. This is confirmed by the magnetically insensitive FFT, which shows no $1 / f$ noise nor any harmonics. The worse sensitivity

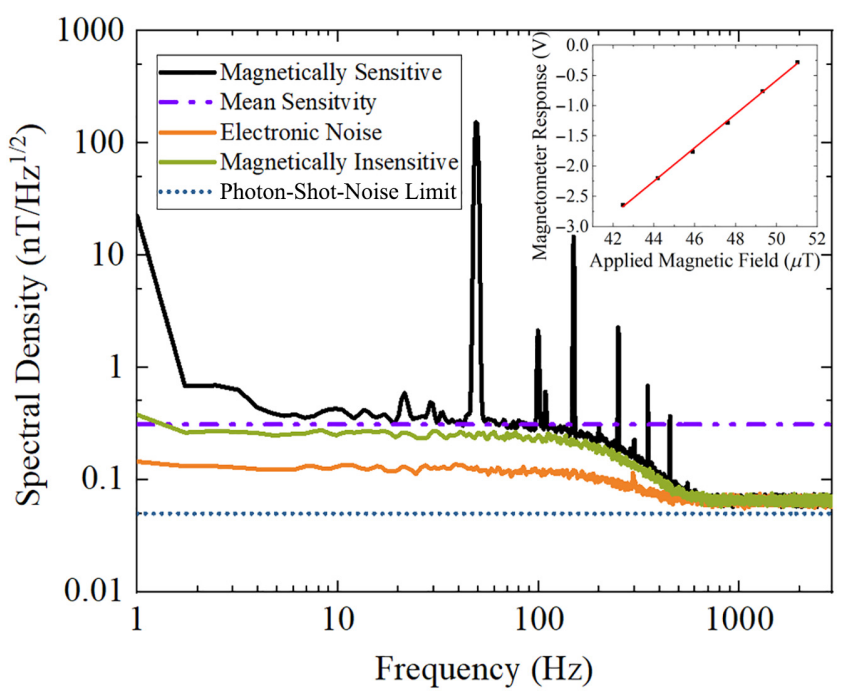

FIG. 3. Diamond magnetometry sensitivity spectrum: the mean sensitivity is $310 \mathrm{pT} / \sqrt{\mathrm{Hz}}$ from $10-150 \mathrm{~Hz}$. The noise floor is shown when no longer on a resonant frequency (magnetically insensitive) and with no applied laser or microwaves (electronic noise). The on resonance, off resonance, and electronic noise are the mean of 1601 -s FFTs. Inset is a figure that demonstrates the magnetometer responsivity for a given applied test field. 
using this method is due to the nonoptimal test field orientation. As the magnetic fields are applied along (100) a reduction in the obtained magnetometer responsivity by a factor of $\cos (109.5 / 2) \sim 0.57$ is expected; we obtain a difference of 0.55 between the two values.

For the targeted application the fields of interest are applied along the (100) direction and thus the sensitivity using the second method is considered to be the true sensitivity. Our sensitivity improves on the value of $35 \mathrm{nT} / \sqrt{\mathrm{Hz}}$ previously obtained with a fiber-coupled NVC magnetometer using applied test fields [23,24].

We estimate the sensing volume to be approximately $600 \mu \mathrm{m} \times 4650 \mu \mathrm{m}^{2}$; this is based on a beam waist of approximately $38 \mu \mathrm{m}$. The photon-shot-noise limit is calculated using Eq. (2) from the fluorescence, which is measured to be $1.2 \times 10^{15}$ photons/s by directly measuring the incident power level on a power meter (Thorlabs PM100D equipped with a power meter head Thorlabs S121C). The linewidth of $1.11 \mathrm{MHz}$ and contrast of $1.76 \%$ are extracted directly from an ODMR spectrum prior to lock-in amplification when exciting all three nitrogen-14 hyperfine resonances. From this it is estimated that the photon-shot-noise limit is $50 \mathrm{pT} / \sqrt{\mathrm{Hz}}$. The improved sensitivity of our fiber-coupled magnetometer is achieved through the employment of more efficient microwave excitation and improved ${ }^{12} \mathrm{C}$-enriched diamond material as well as the lens arrangement in the sensor head. We also investigate the relation of the microwave parameters and the $C / \Delta v$ ratio and how this translates to the LIA voltage output in order to maximize the sensitivity.

\section{DISCUSSION}

Our sensitivity is $310 \mathrm{pT} / \sqrt{\mathrm{Hz}}$ and thus we are a factor of approximately 6 away from the shot-noise limit. We attribute the majority of the noise that prevents us from reaching the photon-shot-noise limit to uncanceled laser and microwave noise. It could be possible to reduce the factor away from shot noise through the implementation of a gradiometer, which could cancel out the remaining noise due to the laser. Furthermore, the reduction of the noise floor when we are magnetically insensitive compared to when magnetically sensitive suggests there is a degree of noise provided by background magnetic fields. A gradiometer would also help alleviate this $[24,47]$. To detect signals for MCG it is estimated that the sensitivity required needs to be over an order of magnitude beyond what we currently achieve $[12,18,48]$.

The biggest limitation of our system is the collection efficiency in which significant improvements are expected as the conversion efficiency of green to red photons is calculated to be $0.03 \%$. Improving this would also improve the excitation efficiency. Due to the high refractive index of diamond $n_{d}=2.42$, the majority of light emitted by the defects will undergo total-internal-reflection and thus will escape through the sides of the diamond [7]. A possible option for improvement is an adaptation of the fluorescence waveguide excitation and collection [49], which reported a 96-fold improvement in the light collected. Another approach would be to surround the diamond with a total-internal-reflection lens to collect light from the diamond sides and focus it toward a small area [50], which would be easier to integrate with our system, leading to an enhancement of 56 in the photon collection when compared to a lossless air objective of 0.55 N.A. This would represent a photon enhancement of approximately 30 for our system and assuming a shot-noise-limited scaling the measured sensitivity would become approximately $60 \mathrm{pT} / \sqrt{\mathrm{Hz}}$.

Ferrite flux concentrators demonstrate a $\times 254$ improvement in the sensitivity for a diamond magnetometer [14] at a cost of degrading the spatial resolution due to concentrating the flux from a large area and directing it toward a diamond. Due to the constraints of our system integrating the design discussed in Ref. [14] is not straightforward and thus the expected enhancement to sensitivity is smaller. A further improvement is expected from the dual-resonance technique [14], which would allow our system to be invariant to temperature fluctuations [51], which is essential for practical applications of our magnetometer. Another way to introduce temperature invariance into our system is the use of double-quantum magnetometry $[52,53]$. This would also be compatible with the use of pulsed schemes such as Ramsey magnetometry, which would offer significant improvements to the sensitivity of a magnetometer compared to continuous-wave excitation schemes [5,9]. However, it should be noted that significantly more laser excitation power and more homogeneous microwave driving fields will be required to realize the potential benefits of Ramsey magnetometry [38,54,55].

\section{CONCLUSION}

In this work a fiber-coupled magnetometer using nitrogen-vacancy centers in diamond that reaches an unshielded sensitivity of $(310 \pm 20) \mathrm{pT} / \sqrt{\mathrm{Hz}}$ over the frequency range of $10-150 \mathrm{~Hz}$ has been presented. The mobility of the system and the compact nature of the sensor head are designed to target the application of magnetocardiography, with the design allowing the active sensing area to be $2 \mathrm{~mm}$ away from the magnetic field source of interest.

All data used in the production of this work is available online [56].

\section{ACKNOWLEDGMENTS}

The authors thank Mareike Herrmann and Luke Johnson for materials processing, Jeanette Chattaway and Lance Fawcett of the Warwick Physics mechanical workshop, and Robert Day and David Greenshields of the Warwick Physics electronics workshop. We are grateful 
for insightful discussions with Matthew Turner, Danielle Braje, John Barry, Jennifer Schloss, Ronald Walsworth, Olga Young, and Junichi Isoya. R.L.P. and G.A.S.'s PhD studentships are cofunded by the EPSRC Centre for Doctoral Training in Diamond Science and Technology (Grant No. EP/L015315/1). G.A.S.'s PhD studentship is additionally supported by Bruker. This work is supported by the EPSRC Quantum Technology Hub NQIT (Networked Quantum Information Technologies - Grant No. EP/M013243/1), QCS (Quantum Computing and Simulation - Grant No. EP/T001062/1) and funding from NICOP (Grant No. N62909-16-1-2111-P00002 - Towards a Picotesla DC Diamond Magnetometer). E.C.N. and W.T. are supported by the Warwick University URSS (Undergraduate Research Support Scheme). B.L.G. is supported by the Royal Academy of Engineering. G.W.M. is supported by the Royal Society.

\section{APPENDIX A: ZERO-CROSSING SLOPE VERSUS MODULATION FREQUENCY}

The variation of the zero-crossing slope as a function of the modulation frequency is shown in Fig. 4. The expected trend of a decrease in the zero-crossing slope for higher modulation frequencies due to the finite repolarization time of the NVC is followed $[5,41,46]$. Despite the continued increase of the zero-crossing slope at progressively lower modulation frequencies, the best sensitivity is achieved at a modulation frequency of $3.0307 \mathrm{kHz}$ (data not shown); we attribute this to an increased susceptibility to noise at particularly low modulation frequencies nearer to dc. The value of the zero-crossing slope in Fig. 4 for a modulation frequency of $3.0307 \mathrm{kHz}$ is $(16.2 \pm 0.1) \mathrm{V} / \mathrm{MHz}$.

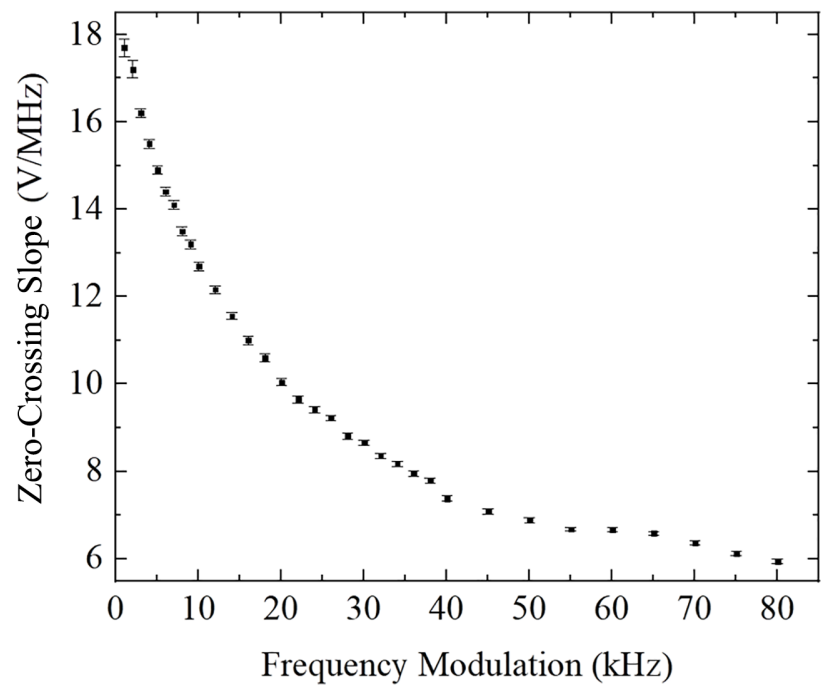

FIG. 4. The zero-crossing slope as a function of the modulation frequency.

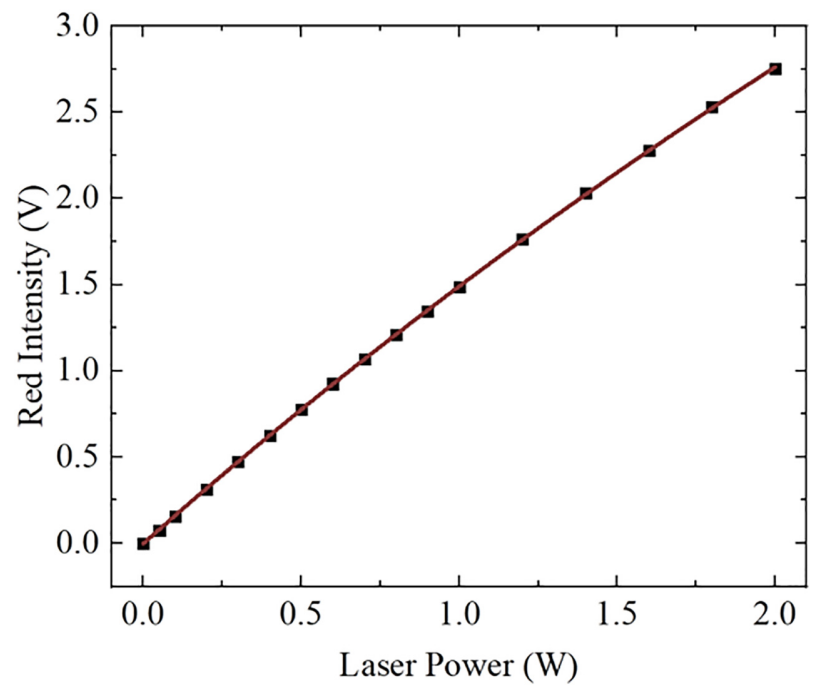

FIG. 5. A power saturation curve plotting the applied laser power against the photodiode output.

Upon further optimization the maximum value of the zerocrossing slope at a modulation of $3.0307 \mathrm{kHz}$ increased to $17.9 \mathrm{~V} / \mathrm{MHz}$. The microwave intensity is not equal for the three frequencies used to excite the hyperfine lines. This means the linewidth of an ODMR resonance depends which microwave excitation(s) created it. This is taken into account during the fitting.

\section{APPENDIX B: POWER SATURATION}

A power saturation measurement is taken with the data, see Fig. 5, fit according to

$$
I=I_{\mathrm{sat}} \frac{P}{P+P_{\mathrm{sat}}},
$$

where $I$ is the fluorescence intensity (in V), $I_{\text {sat }}$ is saturation fluorescence (in V), $P$ is the applied laser power, and $P_{\text {sat }}$ is the saturation power [31]. From this fit it is found that value of $I_{\text {sat }}$ is $(18.8 \pm 0.4) \mathrm{V}$ while $P_{\text {sat }}$ is $(11.6 \pm 0.3) \mathrm{W}$. The regime we are operating at is far from saturation of the NVC defects.

\section{APPENDIX C: DIAMOND CHARACTERIZATION}

The defect concentration within the diamond is determined through Fourier-transform infrared spectroscopy (FTIR) and ultraviolet-visible (UV-vis) spectroscopy. UVvis data, Fig. 6(a), are taken at $80 \mathrm{~K}$ on a Perkin Elmer Lambda 1050 Spectrometer equipped with an Oxford Instruments Optistat cryostat. The concentration is determined to be $4.6 \mathrm{ppm}$ for negatively charged NVC and $0.8 \mathrm{ppm}$ for neutral NVC and is found from the intensities of the 637 and $575 \mathrm{~nm}$ zero-phonon line, respectively [43]. FTIR data, Fig. 6(b), are taken at room temperature 
(a)

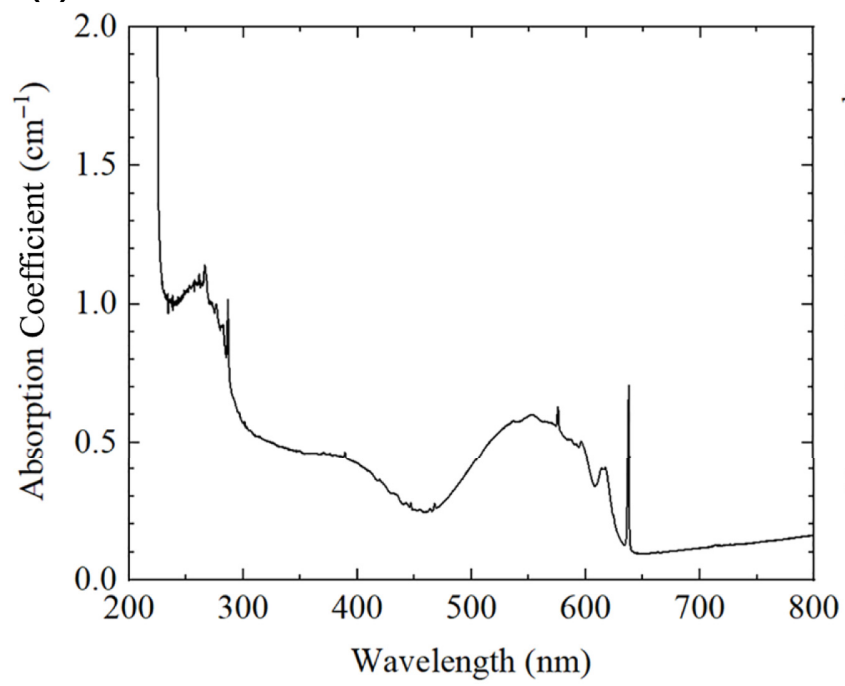

(b)

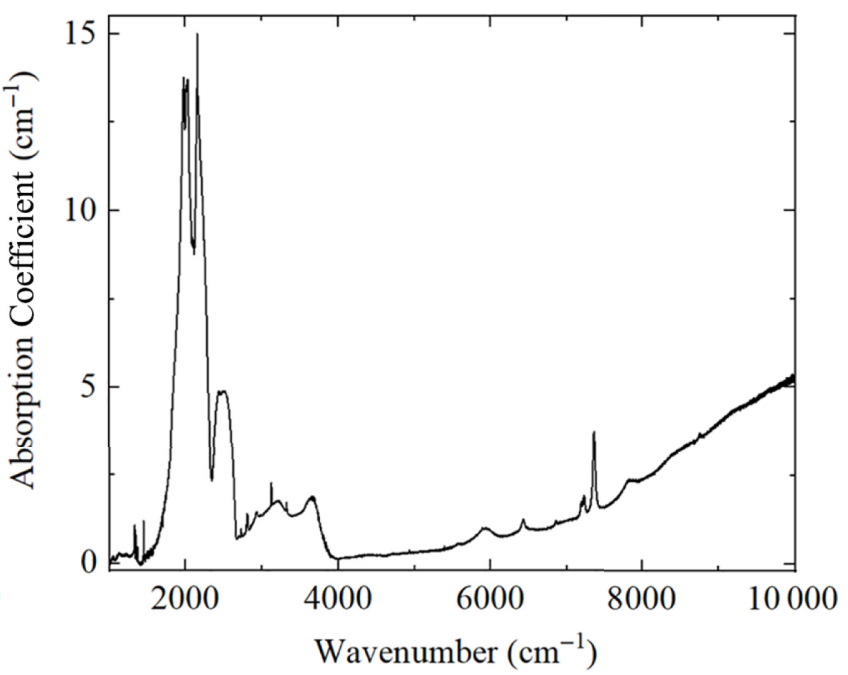

FIG. 6. (a) The UV-vis data to determine the concentrations of neutral and negatively charged NVC. (b) FTIR data to determine the concentrations of neutral and positively charged substitutional nitrogen defects.

using a Perkin Elmer Spectrum GX FT-IR spectrometer. The concentrations from FTIR are established to be $5.6 \mathrm{ppm}$ for neutral substitutional nitrogen $\left(\mathrm{N}_{s}^{0}\right)$ and 3 ppm for positively charged substitutional nitrogen $\left(\mathrm{N}_{s}^{+}\right)$ [44,45]. In addition, EPR studies are performed upon the sample using a Bruker EMX spectrometer equipped with a $90-\mathrm{dB}$ bridge and a Bruker SHQ cavity. The sample concentrations are found to be $(2.8 \pm 0.2)$ ppm negatively charged NVC, $(5.3 \pm 0.5) \mathrm{ppm}$ for neutral substitutional nitrogen and we also identify a concentration of $(1.5 \pm 0.2)$ ppm for negatively charged hydrogen nitrogen vacancy. The discrepancy between the concentrations of negativelycharged NVC obtained through UV-vis and EPR measurements may be due to charge-transfer effects. Pulsed EPR measurements to determine $T_{2}^{*}, T_{2}$ and $T_{1}$ are performed using a Bruker E580 equipped with a MD5 resonator with $T_{2} \sim 1.3 \mu \mathrm{s}$ and $T_{1} \sim 5100 \mu \mathrm{s}$. We estimate a conversion efficiency of approximately $20 \%$ of nitrogen to NVC.

\section{APPENDIX D: SENSOR HEAD}

The use of aluminum for the antenna substrate helps remove heat from the diamond, which mitigates temperature fluctuations in the diamond, which cause shifts of the zero resonance crossing. An image of the sensor head is shown in Fig. 7. To confirm that the temperature of the sensor head is suitable for medical applications, the aluminum temperature is monitored for $1 \mathrm{~h}$ after turning on the laser at a power of $1 \mathrm{~W}$ as shown in Fig. 8. The temperature is recorded using a Fluke TiS PRO Infrared Camera. The error bars are taken as $5 \%$ of the temperature reading recorded by the thermal camera. The sensor head reaches an equilibrium temperature, approximately $26.5^{\circ} \mathrm{C}$, after
20 min making it safe to come into contact with living tissue.

To make the magnetometer fully portable we mount all optics and associated electronics in a rack-mount setup. We use a large heat sink and heat fins to take away heat. However, as there is limited space in the rack, the size of the heat sink is limited. This means that when operating at 2 $\mathrm{W}$ of laser power the laser heats up more than with $1 \mathrm{~W}$. The magnetometry is noisier with $2 \mathrm{~W}$ than with $1 \mathrm{~W}$. We think that the elevated temperature of the GEM laser head rises causing the laser to oscillate between different modes, which introduces noise through mode hopping. Forced air

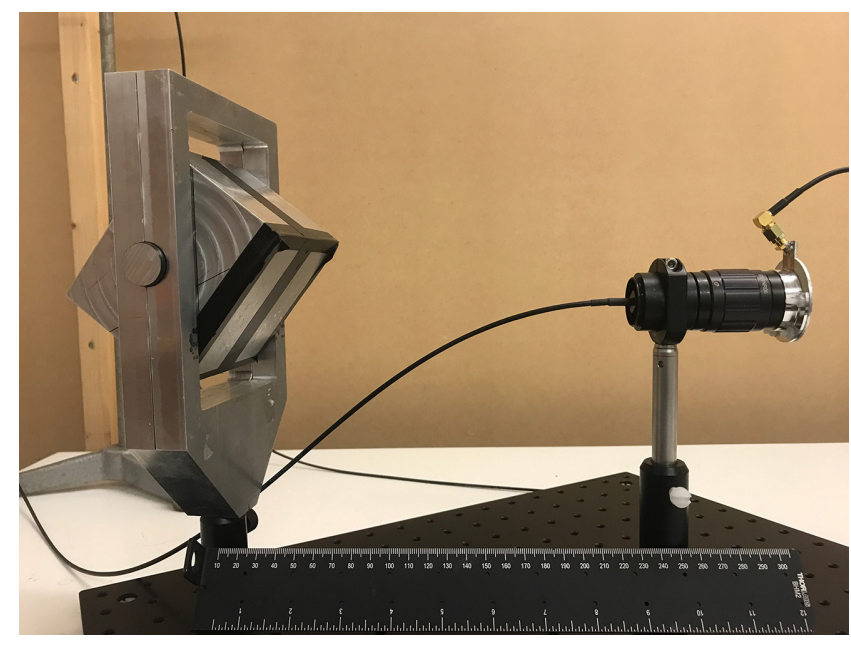

FIG. 7. Picture of the sensor head (black horizontal cylinder in the top right) with a ruler to show the scale size. On the left is a $(\mathrm{Nd}, \mathrm{Fe}) \mathrm{B}$ bias magnet in a rotatable aluminum enclosure. 


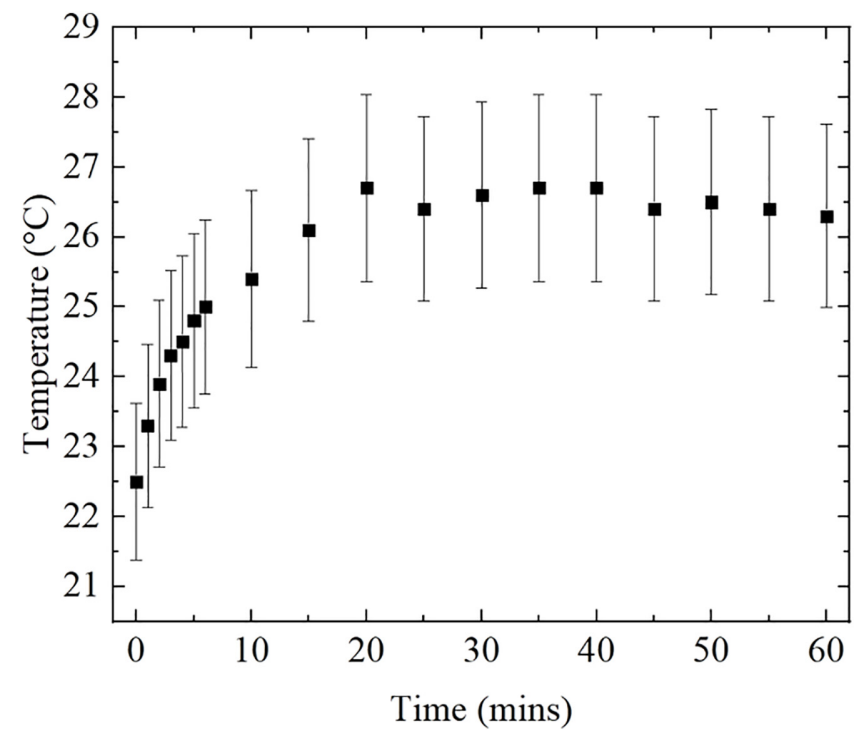

FIG. 8. Temperature variation of the aluminum antenna as a function of time for $60 \mathrm{~min}$.

cooling plates introduce noise and as such passive solutions to heat management are preferred. To operate in a thermally stable regime we choose to use $1 \mathrm{~W}$ of laser power.

[1] G. Balasubramanian, I. Y. Chan, R. Kolesov, M. AlHmoud, J. Tisler, C. Shin, C. Kim, A. Wojcik, P. R. Hemmer, A. Krueger, T. Hanke, A. Leitenstorfer, R. Bratschitsch, F. Jelezko, and J. Wrachtrup, Nanoscale imaging magnetometry with diamond spins under ambient conditions, Nature 455, 648 (2008).

[2] J. M. Taylor, P. Cappellaro, L. Childress, L. Jiang, D. Budker, P. R. Hemmer, A. Yacoby, R. Walsworth, and M. D. Lukin, High-sensitivity diamond magnetometer with nanoscale resolution, Nat. Phys. 4, 810 (2008).

[3] H. Clevenson, L. M. Pham, C. Teale, K. Johnson, D. Englund, and D. Braje, Robust high-dynamic-range vector magnetometry with nitrogen-vacancy centers in diamond, Appl. Phys. Lett. 112, 252406 (2018).

[4] L. M. Pham, D. Le Sage, P. L. Stanwix, T. K. Yeung, D. Glenn, A. Trifonov, P. Cappellaro, P. R. Hemmer, M. D. Lukin, H. Park, A. Yacoby, and R. L. Walsworth, Magnetic field imaging with nitrogen-vacancy ensembles, New J. Phys. 13, 045021 (2011).

[5] J. F. Barry, M. J. Turner, J. M. Schloss, D. R. Glenn, Y. Song, M. D. Lukin, H. Park, and R. L. Walsworth, Optical magnetic detection of single-neuron action potentials using quantum defects in diamond, Proc. Natl. Acad. Sci. 113, 14133 (2016).

[6] J. M. Schloss, J. F. Barry, M. J. Turner, and R. L. Walsworth, Simultaneous Broadband Vector Magnetometry Using Solid-State Spins, Phys. Rev. Appl. 10, 034044 (2018).
[7] D. Le Sage, L. M. Pham, N. Bar-Gill, C. Belthangady, M. D. Lukin, A. Yacoby, and R. L. Walsworth, Efficient photon detection from color centers in a diamond optical waveguide, Phys. Rev. B 85, 121202 (2012).

[8] E. V. Levine, M. J. Turner, P. Kehayias, C. A. Hart, N. Langellier, R. Trubko, D. R. Glenn, R. R. Fu, and R. L. Walsworth, Principles and techniques of the quantum diamond microscope, Nanophotonics 8, 1945 (2019).

[9] J. F. Barry, J. M. Schloss, E. Bauch, M. J. Turner, C. A. Hart, L. M. Pham, and R. L. Walsworth, Sensitivity optimization for $\mathrm{NV}^{-}$diamond magnetometry, Rev. Mod. Phys. 92, 015004 (2020).

[10] E. R. Eisenach, J. F. Barry, L. M. Pham, R. G. Rojas, D. R. Englund, and D. A. Braje, Broadband loop gap resonator for nitrogen vacancy centers in diamond, Rev. Sci. Instrum. 89, 094705 (2018).

[11] L. Bougas, A. Wilzewski, Y. Dumeige, D. Antypas, T. Wu, A. Wickenbrock, E. Bourgeois, M. Nesladek, H. Clevenson, D. Braje, D. Englund, and D. Budker, On the possibility of miniature diamond-based magnetometers using waveguide geometries, Micromachines 9, 276 (2018).

[12] H. Clevenson, M. E. Trusheim, C. Teale, T. Schröder, D. Braje, and D. Englund, Broadband magnetometry and temperature sensing with a light-trapping diamond waveguide, Nat. Phys. 11, 393 (2015).

[13] D. Kim, M. I. Ibrahim, C. Foy, M. E. Trusheim, R. Han, and D. R. Englund, A CMOS-integrated quantum sensor based on nitrogen-vacancy centres, Nat. Electron. 2, 284 (2019).

[14] I. Fescenko, A. Jarmola, I. Savukov, P. Kehayias, J. Smits, J. Damron, N. Ristoff, N. Mosavian, and V. M. Acosta, Diamond magnetometer enhanced by ferrite flux concentrators, Phys. Rev. Res. 2, 023394 (2020).

[15] T. Wolf, P. Neumann, K. Nakamura, H. Sumiya, T. Ohshima, J. Isoya, and J. Wrachtrup, Subpicotesla Diamond Magnetometry, Phys. Rev. X 5, 041001 (2015).

[16] Matthew W. Dale and Gavin W. Morley, Medical applications of diamond magnetometry: Commercial viability, arXiv:1705.01994 (2017).

[17] R. Fenici, D. Brisinda, and A. M. Meloni, Clinical application of magnetocardiography, Expert Rev. Mol. Diagn. 5, 291 (2005).

[18] G. Chatzidrosos, A. Wickenbrock, L. Bougas, N. Leefer, T. Wu, K. Jensen, Y. Dumeige, and D. Budker, Miniature Cavity-Enhanced Diamond Magnetometer, Phys. Rev. Appl. 8, 044019 (2017).

[19] X. Liu, J. Cui, F. Sun, X. Song, F. Feng, J. Wang, W. Zhu, L. Lou, and G. Wang, Fiber-integrated diamond-based magnetometer, Appl. Phys. Lett. 103, 143105 (2013).

[20] I. V. Fedotov, L. V. Doronina-Amitonova, D. A. SidorovBiryukov, N. A. Safronov, A. O. Levchenko, S. A. Zibrov, S. M. Blakley, H. Perez, A. V. Akimov, A. B. Fedotov, P. Hemmer, K. Sakoda, V. L. Velichansky, M. O. Scully, and A. M. Zheltikov, Fiber-optic magnetometry with randomly oriented spins, Opt. Lett. 39, 6755 (2014).

[21] D. Duan, G. X. Du, V. K. Kavatamane, S. Arumugam, Y.-K. Tzeng, H.-C Chang, and G. Balasubramanian, Efficient nitrogen-vacancy centers' fluorescence excitation and collection from micrometer-sized diamond by a tapered optical fiber in endoscope-type configuration, Opt. Express 27, 6734 (2019). 
[22] I. V. Fedotov, L. V. Doronina-Amitonova, D. A. SidorovBiryukov, N. A. Safronov, S. Blakley, A. O. Levchenko, S. A. Zibrov, A. B. Fedotov, S. Ya. Kilin, and M. O. Scully, Fiber-optic magnetic-field imaging, Opt. Lett. 39, 6954 (2014).

[23] S. M. Blakley, I. V. Fedotov, L. V. Amitonova, E. E. Serebryannikov, H. Perez, S. Y. A. Kilin, and A. M. Zheltikov, Fiber-optic vectorial magnetic-field gradiometry by a spatiotemporal differential optical detection of magnetic resonance in nitrogen-vacancy centers in diamond, Opt. Lett. 41, 2057 (2016).

[24] S. M. Blakley, I. V. Fedotov, S. Ya. Kilin, and A. M. Zheltikov, Room-temperature magnetic gradiometry with fiber-coupled nitrogen-vacancy centers in diamond, Opt. Lett. 40, 3727 (2015).

[25] S. Maayani, C. Foy, D. Englund, and Y. Fink, Distributed quantum fiber magnetometry, Laser Photon. Rev. 13, 1900075 (2019).

[26] D. Zheng, Z. Ma, W. Guo, L. Niu, J. Wang, X. Chai, Y. Li, Y. Sugawara, C. Yu, Y. Shi, X. Zhang, J. Tang, H. Guo, and J. Liu, A hand-held magnetometer based on an ensemble of nitrogen-vacancy centers in diamond, J. Phys. D 53, 155004 (2020)

[27] J. L. Webb, J. D. Clement, L. Troise, S. Ahmadi, G. J. Johansen, A. Huck, and U. L. Andersen, Nanotesla sensitivity magnetic field sensing using a compact diamond nitrogen-vacancy magnetometer, Appl. Phys. Lett. 114, 231103 (2019).

[28] A. K. Dmitriev and A. K. Vershovskii, Concept of a microscale vector magnetic field sensor based on nitrogenvacancy centers in diamond, J. Opt. Soc. Am. B 33, B1 (2016).

[29] F. M. Stürner, A. Brenneis, J. Kassel, U. Wostradowski, R. Rölver, T. Fuchs, K. Nakamura, H. Sumiya, S. Onoda, J. Isoya, and F. Jelezko, Compact integrated magnetometer based on nitrogen-vacancy centres in diamond, Diam. Relat. Mater. 93, 59 (2019).

[30] A. Frangeskou, G. Morley, B. Green, B. Breeze, M. Dale, and R. Patel, Defect centre-based sensor, WO 2020/157497 A1 (2020).

[31] N. Aslam, G. Waldherr, P. Neumann, F. Jelezko, and J. Wrachtrup, Photo-induced ionization dynamics of the nitrogen vacancy defect in diamond investigated by singleshot charge state detection, New J. Phys. 15, 013064 (2013).

[32] M. W. Doherty, N. B. Manson, P. Delaney, F. Jelezko, J. Wrachtrup, and L. C. L. Hollenberg, The nitrogenvacancy colour centre in diamond, Phys. Rep. 528, 1 (2013).

[33] V. M. Acosta, E. Bauch, M. P. Ledbetter, C. Santori, K.-M. C. Fu, P. E. Barclay, R. G. Beausoleil, H. Linget, J. F. Roch, F. Treussart, S. Chemerisov, W. Gawlik, and D. Budker, Diamonds with a high density of nitrogen-vacancy centers for magnetometry applications, Phys. Rev. B 80, 115202 (2009)

[34] L. Rondin, J.-P. Tetienne, T. Hingant, J.-F. Roch, P. Maletinsky, and V. Jacques, Magnetometry with nitrogenvacancy defects in diamond, Rep. Prog. Phys. 77, 056503 (2014).

[35] A. Dréau, M. Lesik, L. Rondin, P. Spinicelli, O. Arcizet, J. F. Roch, and V. Jacques, Avoiding power broadening in optically detected magnetic resonance of single NV defects for enhanced dc magnetic field sensitivity, Phys. Rev. B 84, 195204 (2011).

[36] J. A. Van Wyk, E. C. Reynhardt, G. L. High, and I. Kiflawi, The dependences of ESR line widths and spin-spin relaxation times of single nitrogen defects on the concentration of nitrogen defects in diamond, J. Phys. D 30, 1790 (1997).

[37] H. Zhou, J. Choi, S. Choi, R. Landig, A. M. Douglas, J. Isoya, F. Jelezko, S. Onoda, H. Sumiya, P. Cappellaro, H. S. Knowles, Hongkun Park, and M. D. Lukin, Quantum metrology with strongly interacting spin systems, arXiv:1907.10066 [quant-ph] (2019).

[38] E. Bauch, C. A. Hart, J. M. Schloss, M. J. Turner, J. F. Barry, P. Kehayias, S. Singh, and R. L. Walsworth, Ultralong Dephasing Times in Solid-State Spin Ensembles via Quantum Control, Phys. Rev. X 8, 31025 (2018).

[39] G. Balasubramanian, P. Neumann, D. Twitchen, M. Markham, Ro. Kolesov, N. Mizuochi, J. Isoya, J. Achard, J. Beck, J. Tissler, V. Jacques, P. R Hemmer, F. Jelezko, and J. Wrachtrup, Ultralong spin coherence time in isotopically engineered diamond, Nat. Mater. 8, 383 (2009).

[40] T. Kanada, Evaluation of modal noise in multimode fiberoptic systems, J. Lightwave Technol. 2, 11 (1984).

[41] H. A. R. El-Ella, S. Ahmadi, A. M. Wojciechowski, A. Huck, and U. L. Anderson, Optimised frequency modulation for continuous-wave optical magnetic resonance sensing using nitrogen-vacancy ensembles, Opt. Express 25, 14809 (2017).

[42] A. M. Edmonds, et al., Generation of nitrogen-vacancy ensembles in diamond for quantum sensors: Optimization and scalability of CVD processes, arXiv:2004.01746 (2020).

[43] M. W. Dale, Ph.D. thesis, University of Warwick, 2015.

[44] S. C. Lawson, D. Fisher, D. C. Hunt, and M. E. Newton, On the existence of positively charged single-substitutional nitrogen in diamond, J. Phys.: Condens. Matter 10, 6171 (1998).

[45] S. Liggins, Ph.D., University of Warwick, 2010.

[46] R. S. Schoenfeld and W. Harneit, Real Time Magnetic Field Sensing and Imaging Using a Single Spin in Diamond, Phys. Rev. Lett. 106, 030802 (2011).

[47] V. M. Acosta, E. Bauch, A. Jarmola, L. J. Zipp, M. P. Ledbetter, and D. Budker, Broadband magnetometry by infrared-absorption detection of nitrogen-vacancy ensembles in diamond, Appl. Phys. Lett. 97, 174104 (2010).

[48] S. Watanabe and S. Yamada, Magnetocardiography in early detection of electromagnetic abnormality in ischemic heart disease, J. Arrhythm. 24, 4 (2008).

[49] S. Zhang, Z. Ma, L. Qin, Y. Fu, Y. Shi, J. Liu, and Y. J. Li, Fluorescence detection using optical waveguide collection device with high efficiency on assembly of nitrogen vacancy centers in diamond, Appl. Phys. Express 11, 013007 (2018).

[50] L. Xu, H. Yuan, N. Zhang, J. Zhang, G. Bian, P. Fan, M. Li, C. Zhang, Y. Zhai, and J. Fang, High-efficiency fluorescence collection for $\mathrm{NV}^{-}$center ensembles in diamond, Opt. Express 27, 10787 (2019).

[51] V. M. Acosta, E. Bauch, M. P. Ledbetter, A. Waxman, L.-S. Bouchard, and D. Budker, Temperature Dependence 
of the Nitrogen-Vacancy Magnetic Resonance in Diamond, Phys. Rev. Lett. 104, 070801 (2010).

[52] K. Fang, V. M. Acosta, C. Santori, Z. Huang, Kohei M. Itoh, H. Watanabe, S. Shikata, and R. G. Beausoleil, HighSensitivity Magnetometry Based on Quantum Beats in Diamond Nitrogen-Vacancy Centers, Phys. Rev. Lett. 110, 130802 (2013).

[53] H. J. Mamin, M. H. Sherwood, M. Kim, C. T. Rettner, K. Ohno, D. D. Awschalom, and D. Rugar, Multipulse Double-Quantum Magnetometry with Near-Surface Nitrogen-Vacancy Centers, Phys. Rev. Lett. 113, 1 (2014).

[54] S. Ahmadi, H. A. R. El-Ella, J. O. B. Hansen, A. Huck, and U. L. Andersen, Pump-Enhanced Continuous-Wave Mag- netometry Using Nitrogen-Vacancy Ensembles, Phys. Rev. Appl. 8, 034001 (2017).

[55] K. Jensen, N. Leefer, A. Jarmola, Y. Dumeige, V. M. Acosta, P. Kehayias, B. Patton, and D. Budker, Cavity-Enhanced Room-Temperature Magnetometry Using Absorption by Nitrogen-Vacancy Centers in Diamond, Phys. Rev. Lett. 112, 160802 (2014).

[56] R. L. Patel, L. Q. Zhou, A. C. Frangeskou, G. A. Stimpson, B. G. Breeze, A. Nikitin, M. W. Dale, E. C. Nichols, W. Thornley, B. L. Green, M. E. Newton, A. M. Edmonds, M. L. Markham, D. J. Twitchen, and G. W. Morley, Data for: Subnanotesla Magnetometry with a Fiber-Coupled Diamond Sensor, https://wrap.warwick.ac.uk/141730 (2020). 